\title{
ON THE EXISTENCE OF A CONTROL MEASURE FOR STRONGLY BOUNDED VECTOR MEASURES
}

\author{
BY JAMES K. BROOKS ${ }^{1}$
}

Communicated by Bertram Yood, April 29, 1971

In this note we extend a theorem of Bartle, Dunford, and Schwartz [1] which states that for every countably additive measure defined on a $\sigma$-algebra there exists a positive "control measure" $\nu$ such that $\nu(E) \rightarrow 0$ if and only if $\|\mu\|(E) \rightarrow 0$, where $\|\mu\|$ is the semivariation of $\mu$. In this paper, $\mu$, which is defined on a ring $\Sigma$, is assumed to be finitely additive and strongly bounded (s-bounded) [8] (that is $\mu\left(E_{i}\right) \rightarrow 0$ whenever $\left\{E_{i}\right\}$ is a disjoint sequence of sets). The existing decomposition and extension theorems for vector measures can now be easily deduced by using the control measure. These applications will be presented in [2].

$\mathfrak{X}$ is a Banach space over the reals (the complex case is treated in a similar fashion); $S^{*}$ is the unit sphere in the conjugate space of $\mathfrak{X}$. $\sigma(\mathcal{E})$ denotes the $\sigma$-algebra generated by the class of sets $\varepsilon$. A $\delta$-ring is a ring of sets closed under countable intersections.

THEOREM 1. Let $\Sigma$ be a ring of subsets of a set $S . \mu: \Sigma \rightarrow \mathfrak{X}$ is finitely additive and s-bounded if and only if there exists a positive finitely additive bounded set function $\nu$ defined on $\Sigma$ such that

$$
\lim _{\nu(E) \rightarrow 0} \mu(E)=0
$$

and

$$
\nu(E) \leqq \sup \{\|\mu(F)\|: F \subseteq E, F \in \Sigma\}, \quad E \in \Sigma .
$$

Sketch of the proof. First assume $\Sigma$ is an algebra. Let $T$ be the isometric isomorphism of $b a(S, \Sigma)$ onto $b a\left(S_{1}, \Sigma_{1}\right)$, where $\Sigma_{1}$ is the Stone algebra of all open-closed subsets of the compact totally disconnected Hausdorff space $S_{1}$ [4, IV.9]. $U$ is the isometric isomorphism between $b a\left(S_{1}, \Sigma_{1}\right)$ and $c a\left(S_{1}, \Sigma_{2}\right)$, where $\Sigma_{2}=\sigma\left(\Sigma_{1}\right)$.

We prove that $\left\{(U T)\left(x^{*} \mu\right): x^{*} \in S^{*}\right\}$ is uniformly countably additive on $\Sigma_{2}$. It suffices to show that $\left\{(U T)\left[\left(x^{*} \mu\right)^{+}\right]: x^{*} \in S^{*}\right\}$ is uniformly countably additive, where $x^{*} \mu=\left(x^{*} \mu\right)+-\left(x^{*} \mu\right)-$ is the Jordan

AMS 1970 subject classifications. Primary 22A45.

Key words and phrases. Stone algebra, unconditional convergence, weak convergence, weakly compact.

1 This research was supported in partly by NSF Grant GP 28617. 
decomposition of $x^{*} \mu$. Assume the contrary. There exist $A_{i} \in \Sigma_{2}$, $A_{i} \searrow \varnothing, \epsilon>0, x_{i}^{*} \in S^{*}$ such that $\left|\lambda_{i}\left(A_{i}\right)\right|>\epsilon, i=1,2, \cdots$, where $\lambda_{i}=(U T)\left(x_{i}^{*} \mu\right)^{+}$. Let $\Sigma_{01}$ be a countable subalgebra of $\Sigma_{1}$ such that all the $A_{i}$ belong to $\Sigma_{02}=\sigma\left(\Sigma_{01}\right)$. By a diagonal process, we may assume that the $\lambda_{i}$ converge pointwise on $\Sigma_{01}$. Let $\lambda$ denote this limit. By using the unconditional convergence property of $s$-bounded measures $[8,2.3]$, the Orlicz-Pettis theorem, and a result of Procelli [7], we can show that the sequence $\lambda_{i}$ converges weakly to $\lambda$ in $b a\left(S_{1}, \Sigma_{01}\right)$. By a result of Leader [5] this implies that the $\lambda_{i}$ are uniformly absolutely continuous with respect to a positive finitely additive bounded set function $\varphi$ defined on $\Sigma_{01}$. The extensions of the $\lambda_{i}$ to $\Sigma_{02}$ are uniformly absolutely continuous with respect to the extension of $\varphi$ to $\Sigma_{02}$. This yields a contradiction since the $A_{i} \in \Sigma_{02}$. Now since the $\left\{(U T)\left(x^{*} \mu\right)^{+}: x^{*} \in S^{*}\right\}$ are uniformly countably additive and uniformly bounded on $\Sigma_{2}$, the Bartle-Dunford-Schwartz theorem yields a $\nu_{+}^{\prime}$ defined on $\Sigma_{2}$ that acts as a control measure for the family $\left\{(U T)\left(x^{*} \mu\right)^{+}: x^{*} \in S^{*}\right\} . \nu_{-}^{\prime}$ is obtained in a similar fashion. $1 / 2\left[(U T)^{-1}\left(\nu_{+}^{\prime}+\nu_{-}^{\prime}\right)\right]$ yields the desired set function.

Now assume $\Sigma$ is a ring. By analysing the structure of the algebra $\Sigma^{*}$ generated by $\Sigma$, one can prove that $\mu$ can be extended to $\Sigma^{*}$ and that the extension is $s$-bounded on $\Sigma^{*}$. This then reduces to the previous case.

REMARK 1. By means of an example found in [3], one can show that the conclusion of the theorem is false if the assumption of $s$-boundedness is dropped.

In view of the above theorem and the results in [9], we have the following:

Corollary. Let $\mu: \Sigma \rightarrow \mathfrak{X}$ be finitely additive, where $\Sigma$ is a ring. $\mu$ is $s$-bounded if and only if the range of $\mu$ is conditionally weakly compact.

THEOREM 2. Let $\mu: \Sigma \rightarrow \mathfrak{X}$ be countably additive, where $\Sigma$ is a ring. Then there exists a countably additive bounded set function $\nu$ defined on $\Sigma$ which is a control measure for $\mu$ if and only if $\mu$ is s-bounded.

Sketch of PROof. If $\mu$ is $s$-bounded, then by Theorem 1 there exists a bounded finitely additive control measure $\nu$. The conclusion in Theorem 1 implies that $\nu(E) \leqq\|\mu\|(E)=\sup \{\|\mu(F)\|: F \subseteq E\}$. One can show that since $\mu$ is countably additive, $E_{i} \searrow \varnothing$ implies that $\|\mu\|\left(E_{i}\right) \rightarrow 0$; this in turn implies that $\nu$ is countably additive.

REMARK 2. Due to a recent result of Rybakov, one can choose the control measures appearing in Theorems 1 and 2 to be the total variation function of the measure $x^{*} \mu$, for some $x^{*} \in \mathfrak{X}^{*}$.

REMARK 3. Using Zorn's lemma, the control measures can be chosen 
to be dominated by $\|\mu\|$ and also be maximal with respect to the usual order defined on set functions.

REMARK 4. For the bounded case this answers the question posed by Dinculeanu and Kluvanek [3, p. 505] concerning the existence of control measures for vector measures defined on $\delta$-rings. If one requires that $\nu$ be only finite valued and not bounded, then $\mathrm{S}$. Ohba has shown [6] that $\nu$ exists, without the requirement of $s$-boundedness of $\mu$, when $\mathfrak{X}$ is separable. Examples show that without the separability condition the result is false.

We say that a family $\Gamma$ of vector measures is uniformly $s$-bounded if $\mu\left(E_{i}\right) \rightarrow 0$ uniformly for $\mu \in \Gamma$, whenever $\left\{E_{i}\right\}$ is a disjoint sequence of sets. This concept is equivalent to uniform countable additivity when the measures are countably additive on a $\sigma$-ring. The technique in the proof of Theorem 1 is used to prove the following theorem.

THEOREM 3. Let $\Sigma^{*}$ be the $\sigma$-ring generated by the ring $\Sigma$. If a uniformly bounded family of countably additive vector measures is uniformly s-bounded on $\Sigma$, then the family is uniformly countably additive on $\Sigma^{*}$.

The following theorem contains a converse to the Nikodym theorem. The proof uses the above theorem and Lemma IV. 8.8 in [4].

THEOREM 4. Let $\left\{\mu_{n}\right\}$ be a uniformly bounded sequence of countably additive vector measures defined on a $\sigma$-ring $\Sigma^{*}$ generated by the ring $\Sigma$ such that for every $E$ in $\Sigma \lim _{n} \mu_{n}(E)$ exists. Then $\lim _{n} \mu_{n}\left(E^{*}\right)$ exists for every $E^{*}$ in $\Sigma^{*}$ if and only if $\left\{\mu_{n}\right\}$ is uniformly s-bounded on $\Sigma$.

\section{REFERENCES}

1. R. G. Bartle, N. Dunford and J. T. Schwartz, Weak compactness and vector measures, Canad. J. Math. 7 (1955), 289-305. MR 16, 1123.

2. J. K. Brooks and H. Walker, On strongly bounded vector measures and applications to extensions, decompositions and weak compactness, Rev. Roumaine Math. Pures Appl. (to appear).

3. N. Dinculeanu and I. Kluvánek, On vector measures, Proc. London Math. Soc. (3) 17 (1967), 505-512. MR 35 \#5571.

4. N. Dunford and J. T. Schwartz, Linear operators. I: General theory, Pure and Appl. Math., vol. 7, Interscience, New York, 1958. MR 22 \#8302.

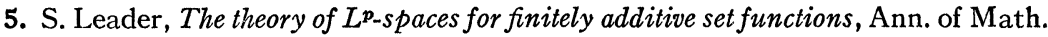
(2) 58 (1953), 528-543. MR 15, 326.

6. S. Ohba, Correction to "On vector measures. I", Proc. Japan Acad. (to appear).

7. P. Porcelli, Two embedding theorems with applications to weak convergence and compactness in spaces of additive type functions, J. Math. Mech. 9 (1960), 273-292. MR 23 \#A2034.

8. C. E. Rickart, Decomposition of additive set functions, Duke Math. J. 10 (1943), 653-665. MR 5, 232.

9. J. J. Uhl, Jr., Extensions and decompositions of vector measures, Proc. London Math. Soc. (to appear).

University of Florida, Gainesville, Florida 32601 